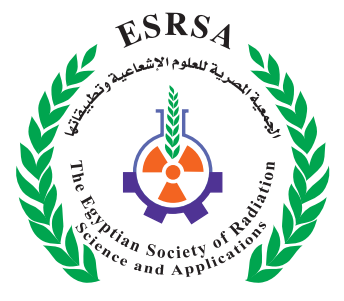

\title{
Modeling and Simulation of Proton Beam Therapy by using Geant4/GATE
}

\author{
Ibrahim, H.F.; Musa, E.S. ${ }^{1}$ and Attalla, E.M. ${ }^{2}$
}

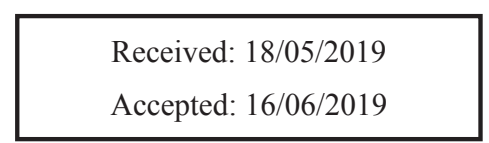

E-mail: elafmousa94@gmail.com

\section{KEYWORDS}

Proton Therapy,

Range Modulator,

Bragg Peak, Monte

Carlo Simulation.

\section{ABSTRACT}

Proton Therapy is one of the most advanced cancer radiotherapy techniques. It offers more accurate dose deposition than conventional radiotherapy techniques, and therefore requires convenient procedures for quality assurance. In this technique, a Spread Out Bragg Peak (SOBP) is produced to establish a uniform dose distribution over the tumor volume. In order to produce a SOBP, several pristine Bragg peaks of different entrance energies and hence different ranges, with certain intensities (weights) should be combined together. In a passive beam scattering system, a mono-energetic pencil beam is extracted from an accelerator, and its diameter is modified by different scattering sheets. Next, a SOBP is produced by using energy modulation devices such as a Range Modulator Wheel (RMW) consisting of steps of variable thicknesses and angles, or by using a ridge filter. In this work, the Geant4/GATE application was used to simulate a typical passive scattering beamline system. To validate the developed model, the CATANA transport beamline of the INFNLNS in Catania, Italy, was simulated in GATE. A set of pristine Bragg peaks were obtained from the MC simulations by using PMMA range shifters of different thicknesses. A mathematical algorithm was used with the simulated pristine dose profiles as its inputs, in order to calculate the weight of each pristine Bragg peak, and hence to obtain the required angular span of each RMW step. As a result, a SOBP was produced to

1. Physics Department, Faculty of Science, Cairo University.

2. Radiation Oncology Department, National Cancer Institute, Cairo University. 
ensure a flat dose distribution in a water phantom. Once the designed RMW was realized, the simulated dose distribution from a $62.8 \mathrm{MeV}$ proton beam was compared successfully with the experimental results from the CATANA facility. Other RMWs which correspond to different entrance energies $(50,60,70$, 80,90 and $100 \mathrm{MeV}$ ) useful for the treatment of eye and brain tumors were newly designed and tested in this study to demonstrate the valid energy range of this technique. Multiple RMWs were mathematically designed to produce plateau sizes at nearly $20 \%$, $40 \%$ and $60 \%$ of the total depth of the original proton beam Bragg peaks.

\section{INTRODUCTION}

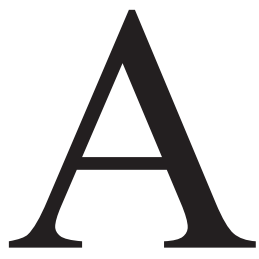

ccording to the World Health Organization (WHO), "cancer is considered to be the major cause of death worldwide, with an estimated number of deaths equals 9.6 million" in 2018 (WHO, 2018). Cancer starts when changes of genes take place within a cell or a group of normal cells. Later, one or few of these cells begin to grow and divide in an uncontrolled way making tumors. These primary tumors sometimes spread to other parts of the body and form secondary tumors. There are more than 100 different types of cancers, such as brain, lung, and prostate cancers, leukemia and ocular melanoma (Cancercenter, 2019).

Scientists believe that cancer results from the interaction of many factors. These factors may be genetic, environmental, or harmful habits of individuals such as smoking (Parkin, 2005). Depending on its type and how advanced it is, cancer can be treated with different techniques or combinations of them, such as Chemotherapy, Surgery, and Radiotherapy. In this study, we focused on one of the advanced radiotherapy techniques, the proton beam therapy, which demonstrates superiority over other conventional radiotherapy methods such as X-ray. In proton therapy, a beam of protons with energy in the range of $50-300 \mathrm{MeV}$ is directed towards the tumor. The unique characteristics of how protons interact with matter allow them to deliver accurate therapeutic radiation doses, while reducing doses to healthy tissues and organs, resulting in fewer complexity and bad side effects than conventional radiotherapy techniques (Yajnik, 2013).

Monte Carlo (MC) transport techniques are used increasingly in radiation therapy. The interest in these methods has grown due to the need for accurate numerical techniques, to solve many of radiation transport problems that arise in radiation therapy, medical imaging, and nuclear medicine. With the rapid progress in hadron therapy, interest has been increasing in understanding the biological effects of radiation, where the MC methods have proven to be an essential tool (Vassiliev, 2017). MC is more accurate than analytical models because it takes into account the physical processes during particle transport. MCNPX, FLUKA, Geant4 (GEometry ANd Tracking version 4) (Agostinelli, 2003) and the Geant4-based GATE (Geant4 Application for Tomographic Emission) (Visvikis, 2006) are the most commonly used general purpose MC codes for proton therapy research.

GATE is a more versatile MC simulation application than other platforms. It has the ability to simulate medical imaging, radiotherapy and dosimetry in the same environment. It can be used to accurately simulate the electromagnetic (EM) and hadronic interactions of radiation particles with matter. In this work, the GATE application was verified to be a good candidate for the simulation of proton therapy beamline systems. The physics model of proton therapy used in simulation techniques is based on the well-known Beth Bloch formula. For a heavy particle with charge $e z$ travelling a distance $X$ at velocity $v$, and energy $E$ into a target with electron number density $n$, the energy loss rate can be approximately expressed as (Nikjoo, 2012): 
$-\frac{d E}{d X}=\frac{4 \pi e^{4} z^{2} n Z}{m_{o} v^{2}} \times \ln \frac{2 m_{o} v^{2}}{I_{\text {avg }}}$

where $m_{0}$ is the rest mass of the electron, $Z$ is the atomic number of the stopping material and $I_{\text {avg }}$ is the mean excitation potential. This formula shows that when the particle velocity decreases, the energy deposition rate increases.

In recent years, there has been a massive progress in the development of proton therapy facilities in many countries around the world such as the USA, Japan and Germany. Other countries already plan to build more new facilities like Russia, Australia and Egypt. According to the Particle Therapy Co-Operative Group (PTCOG) data, about 57 operating proton accelerators worldwide are being used for therapy including facilities in scientific research institutions (PTCOG, 2019).

In this work, a Range Modulator Wheel (RMW) was designed to simulate the CATANA proton therapy center at the Istituto Nazionale di Fisica Nucleare-Laboratori Nazionali del Sud (INFN-LNS) in Catania, Italy. In this center, a $62.8 \mathrm{MeV}$ proton beam, produced by a superconducting cyclotron, is used for the treatment of shallow tumors, like those of the ocular region. The CATANA facility uses the passive scattering method to broaden the beam laterally and longitudinally, to cover the whole target volume. Various mechanical components are located along the beamline to reach this goal. The beamline has a double scattering system with a central stopper to spread out the beam laterally and to reduce the central component of the beam having a Gaussian shape. Based on this original beamline, other RMWs which correspond to different entrance energies (50, $60,70,80,90$ and $100 \mathrm{MeV}$ ) useful for the treatment of eye and brain tumors with different volumes and depths were newly designed and tested successfully in this study. The resulting plateau sizes of the Spread Out Bragg Peaks (SOBPs) covered nearly $20 \%, 40 \%$ and $60 \%$ of the total range for the original proton beam energies.

\section{MATERIALS AND METHODS}

\section{Experimental Setup}

The components of the original CATANA beamline are shown in Fig. 1a, while Fig. 1b shows the actual RMW that was used to produce the SOBP. A $62.8 \mathrm{MeV}$ proton beam, passing through the beamline components was used in the calculations of the deposited doses from the SOBP. The experimental measurements used in this study were taken from published data (Bijan, 2016).

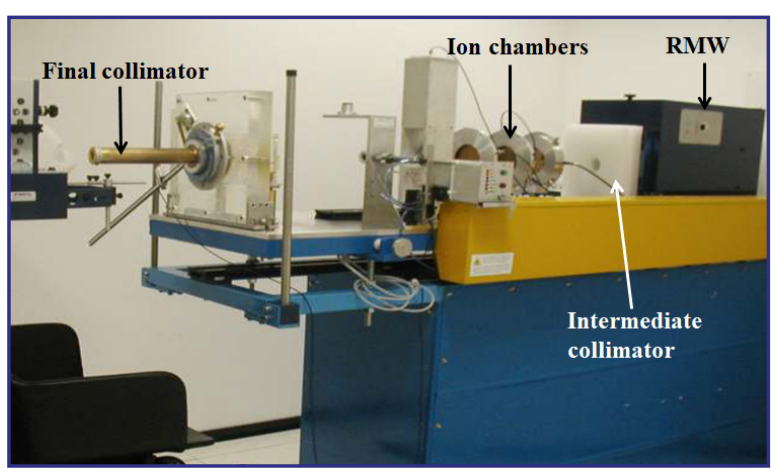

(a) Beamline

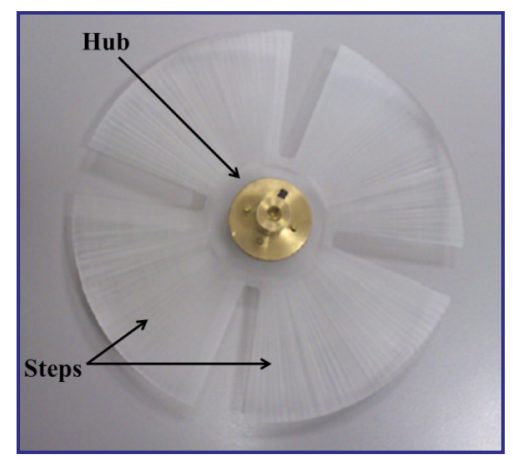

(b) RMW

Fig. (1): CATANA facility.

\section{Simulation Method}

In this study, the simulations to calculate the relative dose values were performed by using different energies of the proton beam $(\leq 100 \mathrm{MeV})$ that could be delivered in facilities similar to CATANA. The simulations were done by GATE using the standard EM model because it is faster and more efficient in computation than the other available EM models in Geant4. In addition, the hadronic model using option 
3 in Geant4 (Geant4) was also used. The full analysis to select the best Geant4 physics models and parameters was performed previously by Grevillot $\boldsymbol{e t}$ al. (2010).

The simulation process was divided into two different parts. The first part was the validation of the SOBP doses produced by the RMW used in the original CATANA facility. The dose voxel (dosel) size was set to be $40 \times 40 \times 0.1 \mathrm{~mm}^{3}$ for the validation step. The second part focused on the designing of new RMWs suitable for several different proton energies in any similar facilities. In order to perform the simulations in GATE accurately, the most important components of the system had to be defined. The original source of protons was simulated as $10 \mathrm{k}$ events/sec to reduce the dose uncertainty below $5 \%$. The ROOT application (Brun, 1997) was used in the final analysis of the deposited doses from the simulations.

\section{Beamline}

The geometry of the CATANA beamline was simulated as precisely as possible, based on the specifications in Table 1. All physical volumes were coupled together following a hierarchical structure. Each volume was characterized by its shape, size, position, and material composition. The base of the structure was represented by the "world" volume. The "world" volume represents a box centered at the origin, with size large enough to include the whole simulation geometry. When any particle escapes from the world volume boundaries, the tracking of this particle stops. The "world" contains other subvolumes referred to as daughter volumes and every daughter volume has a specific purpose and name. The simulated RMW was made out of 16 PMMA steps with thickness $0.9 \mathrm{~mm}$. Finally, a suitable water phantom was used to calculate the proton dose values. Fig. 2a shows the different simulated components of the CATANA beamline, and Fig. 2b shows the simulated RMW used for the validation in GATE.

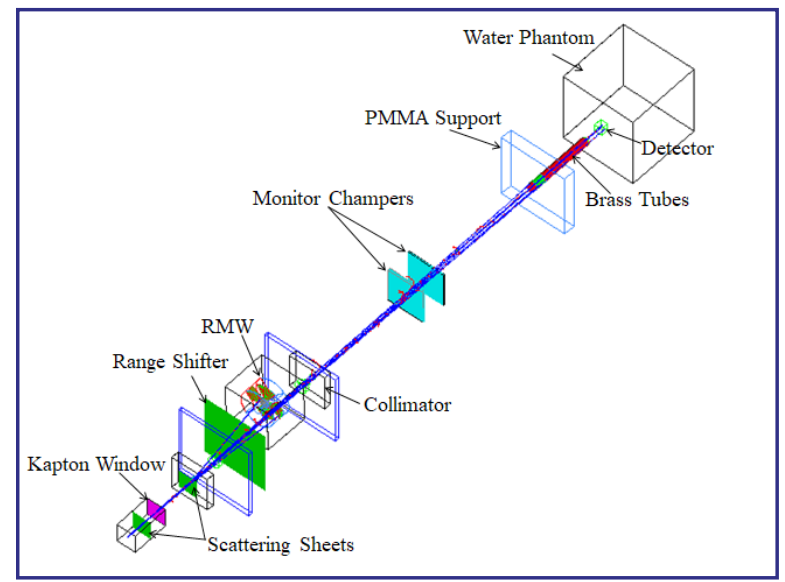

(a) Beamline

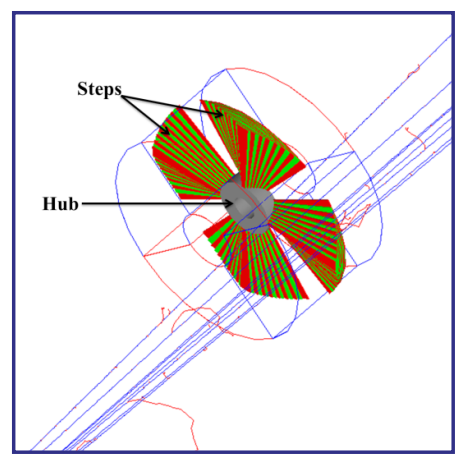

(b) RMW

Fig. (1): Monte Carlo simulations.

\section{Design of New RMWs}

The SOBP is obtained by superimposing different pristine Bragg peaks together. In order to make a new RMW from scratch, the specific weight of each pristine peak should be determined, to convert it to angular span for each different RMW step. In this work, the weights of the single peaks were calculated using a mathematical algorithm (Bijan, 2016). The pristine dose profiles that were obtained through auxiliary range shifters (RMW steps) were used as inputs for this algorithm.

In the CATANA beamline, the RMW is placed $2.050 \mathrm{~m}$ upstream of the isocenter. Dose values for all the pristine depth profiles corresponding to different step thicknesses of the RMW were determined at each peak position. The dose modulation of interest requires 
a number of $N$ points corresponding to peak positions of the pristine profiles, and $D_{i j}(i, j=1,2, \ldots, N)$ refers to the dose of profile $j$ in the peak position $i$. To create a flat dose in the region of the SOBP, the overall dose due to all pristine profiles must be equal to a fixed value $D_{0}$. A system of $N$ coupled linear equations was formed:

$W_{1} D_{i 1}+W_{2} D_{i 2}+W_{3} D_{i 3}+\cdots+W_{N} D_{i N}=D_{i 0}$

in which, the $W_{j}$ parameters are the required weights for each pristine peak. This system of linear equations was solved by the Gauss-Jordan elimina- tion method (Grcar, 2011). The calculated weights were then converted into angular spans.

Using various auxiliary PMMA range shifters as substitutes for the desired RMW steps, a set of pristine Bragg peaks were obtained from simulations. To have an entirely flat modulation region, the thicknesses of RMW range shifters should be chosen in such a way that the distance between neighboring Bragg peaks is similar to the sigma at the distal region of dose profiles. In this work, the peak width in water for each energy, $t_{w}$, was chosen at $80 \%$ of the peak maximum.

Table (1) : Beamline Components.

\begin{tabular}{|c|c|c|c|c|}
\hline Components & Shape & Material & $\begin{array}{c}\begin{array}{c}\text { Length or Thickness } \\
(\mathrm{cm})\end{array} \\
\end{array}$ & Position (cm) \\
\hline Vacuum Pipe & Box & Galactic & 20.000 & 301.000 \\
\hline First Scattering Foil & Box & Tantalum & 0.0015 & 301.000 \\
\hline Kapton Window & Box & Kapton & 0.002 & 291.001 \\
\hline Stopper & Cylinder & Brass & 0.700 & 270.500 \\
\hline Second Scattering Foil & Box & Tantalum & 0.0025 & 270.148 \\
\hline First Collimator & Box & PMMA & 4.000 & 267.300 \\
\hline Modulator Box & Box & Aluminum & 10.000 & 252.300 \\
\hline Range Shifter & Box & PMMA & 0.448 & 239.300 \\
\hline Second Collimator & Box & PMMA & 4.000 & 190.000 \\
\hline \multirow{4}{*}{ First Monitor Chamber } & \multirow{4}{*}{ Box } & Kapton & 0.905 & 126.247 \\
\hline & & Copper & $2.2 \times 10^{-6}$ & 125.797 \\
\hline & & Air & 0.900 & 126.247 \\
\hline & & Copper & $2.2 \times 10^{-6}$ & 126.697 \\
\hline \multirow{4}{*}{$\begin{array}{l}\text { Second Monitor } \\
\text { Chamber }\end{array}$} & \multirow{4}{*}{ Box } & Kapton & 0.905 & 113.142 \\
\hline & & Copper & $2.2 \times 10^{-6}$ & 112.692 \\
\hline & & Air & 0.900 & 113.142 \\
\hline & & Copper & $2.2 \times 10^{-6}$ & 113.592 \\
\hline Nozzle Support & Box & PMMA & 5.900 & 39.750 \\
\hline \multirow{3}{*}{ Brass Tube } & \multirow{3}{*}{ Cylinder } & \multirow{3}{*}{ Brass } & 1.800 & 43.700 \\
\hline & & & 1.800 & 43.700 \\
\hline & & & 1.800 & 20.950 \\
\hline Final Collimator & Cylinder & Brass & 0.700 & 8.350 \\
\hline Detector & Box & Water & 4.000 & -2.000 \\
\hline Phantom & Box & Water & 40.000 & -20.000 \\
\hline
\end{tabular}


The PMMA numbers of steps for each RMW were determined as following:

- Using a suitable database (NIST, 2018), the energy corresponding to the air pristine peak projected range was determined. Small energy loses $\sim 3-4 \mathrm{MeV}$, due to the path of the beam through the system components, were taken into account for precise calculations. The stopping power was also determined for PMMA, $s_{m}$, and water, $s_{w}$.

- The PMMA equivalent thickness $t_{m}$ was obtained by using the relation:

$$
t_{m}=\frac{\rho_{w}}{\rho_{m}} \times \frac{s_{w}}{s_{m}} \times t_{w}
$$

where $\rho_{w}$ and $\rho_{m}$ are the densities of water and the PMMA material respectively.

- The maximum depths were divided by the $t_{m}$ value to determine how many steps are needed to produce the required SOBP sizes to cover $20 \%$, $40 \%$ and $60 \%$ of the original range which correspond to different tumor volumes.

\section{Gamma Index Criteria}

All the SOBP calculations were normalized to the maximum dose in order to make the comparisons in relative doses not in absolute doses. The comparison between the simulation and the measurement results for $62.8 \mathrm{MeV}$ was presented in terms of the gamma index method (Low, 1998), which indicates the accuracy of the simulation results.

The gamma index is a quantitative evaluation method that directly compares the values of the measurements and calculations of the dose distribution. These criteria take into account both the dose difference and the distance-to-agreement (DTA) comparisons. The dose difference represents a point-topoint comparison of the doses from the calculated and measured data, while the DTA is the distance between a measured point and the nearest one in the calculated dose distribution that has a similar dose.
The generalized gamma index function is given by:

$$
\Gamma\left(\vec{r}_{m}, \vec{r}_{s}\right)=\sqrt{\frac{r^{2}\left(\vec{r}_{m}, \vec{r}_{s}\right)}{\Delta d_{M}^{2}}+\frac{\delta^{2}\left(\vec{r}_{m}, \vec{r}_{s}\right)}{\Delta D_{M}^{2}}}
$$

where $\vec{r}_{m}$ is the position vector of the measured points, $\vec{r}_{s}$ is the vector position of the calculated points by simulation, $\Delta d_{M}$ is the DTA criterion (3mm) and $\Delta D_{M}$ is the dose difference criterion (3\%). Here, $r\left(\vec{r}_{m}, \vec{r}_{s}\right)=\left|\vec{r}_{s}-\vec{r}_{m}\right|$ is the distance between the simulation and measured points, and $\delta\left(\vec{r}_{m}, \vec{r}_{s}\right)=D_{s}\left(\vec{r}_{s}\right)-D_{m}\left(\vec{r}_{m}\right)$ is the difference between dose values of the simulated and measured points. The $\gamma$-index is then given by:

$\gamma\left(\vec{r}_{m}\right)=\min \left\{\Gamma\left(\vec{r}_{m}, \vec{r}_{s}\right)\right\} \forall\left\{\vec{r}_{s}\right\}$

Therefore, the calculation passing criteria for each measured point is given by $\gamma\left(\vec{r}_{m}\right) \leq 1$. If $\gamma\left(\vec{r}_{m}\right)>1$, the calculation fails to pass the gamma index criteria.

\section{RESULTS AND DISCUSSION}

\section{Model Validation}

The use of a range shifter is necessary in most common clinical arrangements. In this work the pristine profiles used for the weight computation (Fig. 3a) already include the effect of the range shifter to produce a uniform SOBP. Fig. $3 b$ shows the comparison between the experimental and simulation results by using the gamma index criteria. The percentage of the number of points that passed the criteria (gamma passing rate) was about $98 \%$. The simulated SOBP plateau covered about $67.2 \%$ of the total range $(15.7 \mathrm{~mm})$ for the $62.8 \mathrm{Mev}$ proton beam. Very good horizontal SOBP plateau was deduced from the small magnitude of the slope $(\sim 0.05 \% / \mathrm{mm})$, the flatness of the SOBP was also very good based on the resulting value of the reduced $\chi^{2}$ test for the plateau which was much smaller than $1 \%(\sim 0.015 \%)$.

The specifications of the newly designed RMW are given in Table 2. Using the Mathematical algorithms mentioned above, the weights for all the 
RMW steps were calculated and converted into angular spans. The simulation and experimental results of SOBP, using a $4.48 \mathrm{~mm}$ thick range-shifter, are shown in Fig. $3 b$.

Table (2) : Simulated RMW.

\begin{tabular}{|c|c|c|c|}
\hline Step Number & Thickness $(\mathbf{m m})$ & Relative Weight & Spanning Angle (deg.) \\
\hline 1 & 0.0 & 0.9001 & 18.9896 \\
\hline 2 & 0.9 & 0.4037 & 8.5166 \\
\hline 3 & 1.8 & 0.2972 & 6.2705 \\
\hline 4 & 2.7 & 0.2587 & 5.4585 \\
\hline 5 & 3.6 & 0.2447 & 5.1630 \\
\hline 6 & 4.5 & 0.2428 & 5.1218 \\
\hline 7 & 5.4 & 0.1631 & 3.4416 \\
\hline 8 & 6.3 & 0.1905 & 4.0191 \\
\hline 9 & 7.2 & 0.1987 & 4.1917 \\
\hline 10 & 8.1 & 0.2238 & 4.7227 \\
\hline 11 & 9.0 & 0.1159 & 2.4459 \\
\hline 12 & 9.9 & 0.1789 & 3.7750 \\
\hline 13 & 10.8 & 0.1846 & 3.8945 \\
\hline 14 & 11.7 & 0.2294 & 4.8406 \\
\hline 15 & 12.6 & 0.1193 & 2.5162 \\
\hline 16 & 13.5 & 0.3144 & 6.6326 \\
\hline
\end{tabular}

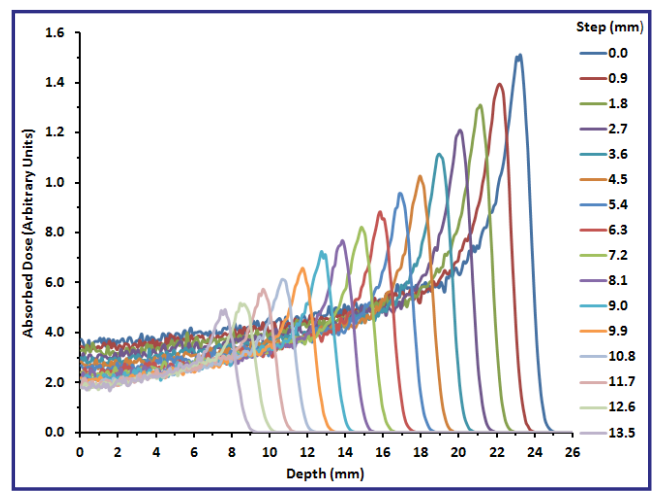

(a) Pristine dose profiles

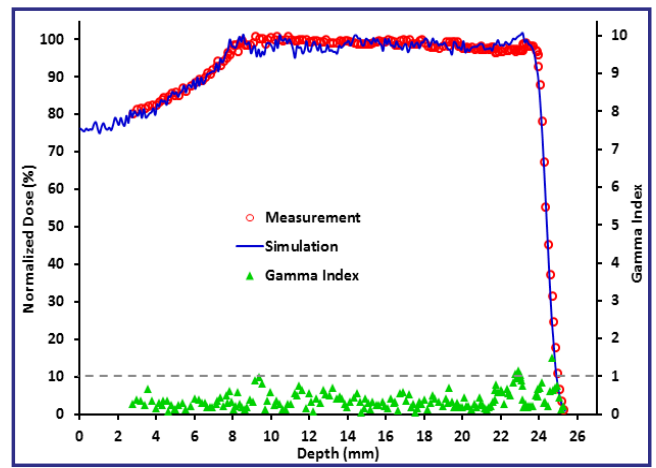

(b) Simulation and experiment results

Fig. (3): Validation Results.

\section{New Proton Energies}

Different RMWs were newly designed for every new proton beam energy and plateau size, 18 RMWs in total. The step thicknesses and angular spans were determined according to the discussed mathematical methods. Fig. 4 (a-f) shows the normalized SOBPs resulting from these RMWs for the plateau sizes of $20 \%, 40 \%$ and $60 \%$, and for different entrance energies of 50,60, 70, 80, 90 and $100 \mathrm{MeV}$ respectively. The number of steps for each RMW, the plateau start and end points, and the plateau sizes are listed in Table 3.

A linear fit was performed for each SOBP in ROOT to determine the horizontalness (slope) and the flatness or "goodness of fit" (reduced $\chi^{2}$ ) of the plateaus. The calculated magnitudes of the plateau slopes are smaller than $0.25 \% / \mathrm{mm}$ which indicates very good horizontal SOBPs for all the studied proton energies and plateau sizes. The flatness of the SOBPs for all energies is also very good based 
on the resulting values of the reduced $\chi^{2}$ from the plateau linear fits which are much smaller than $1 \%$ $(\sim 0.01 \%)$.

The actual plateau sizes were compared with the designed values by using the percentage errors. Ac-

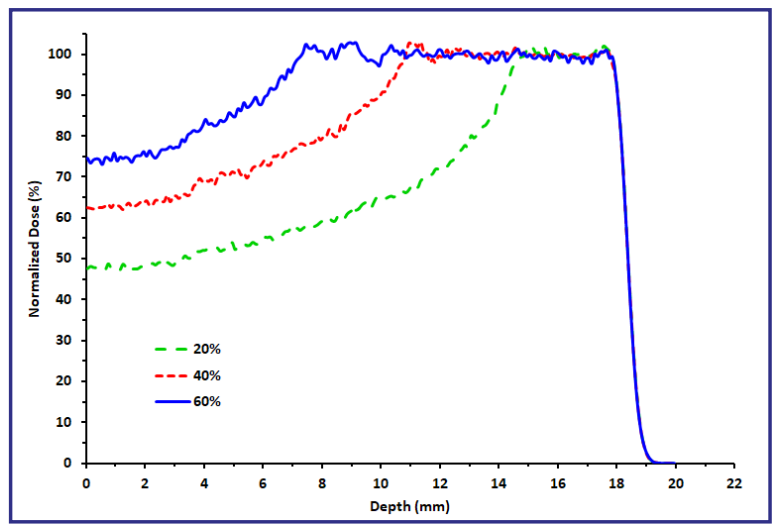

(a) $50 \mathrm{MeV}$

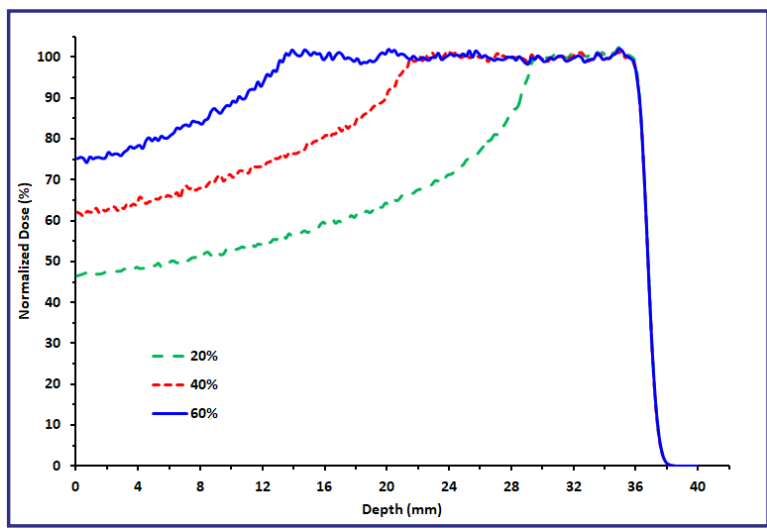

(c) $70 \mathrm{MeV}$

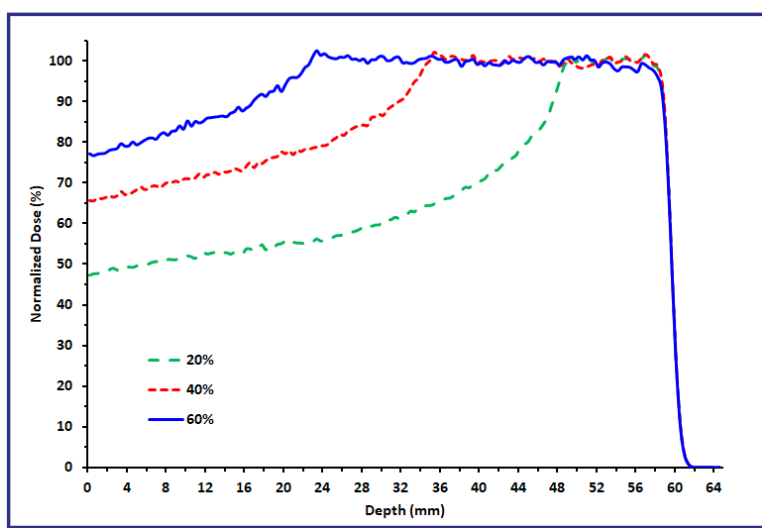

(e) $90 \mathrm{MeV}$ ceptable agreements were observed for the $40 \%$ and $60 \%$ plateaus for all energies. More variations were noted for the $20 \%$ plateaus. This could be due to the small number of steps used for the $20 \%$ plateaus which produced more sharp peaks due to the lower energy absorption in the steps.

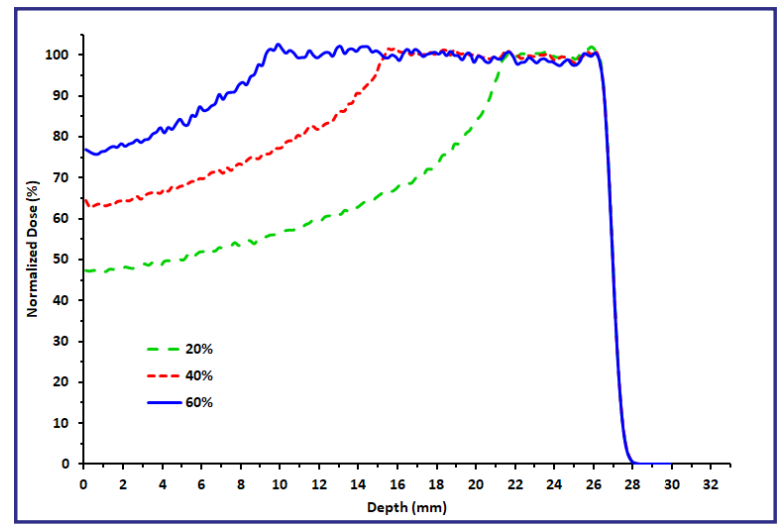

(b) $60 \mathrm{MeV}$

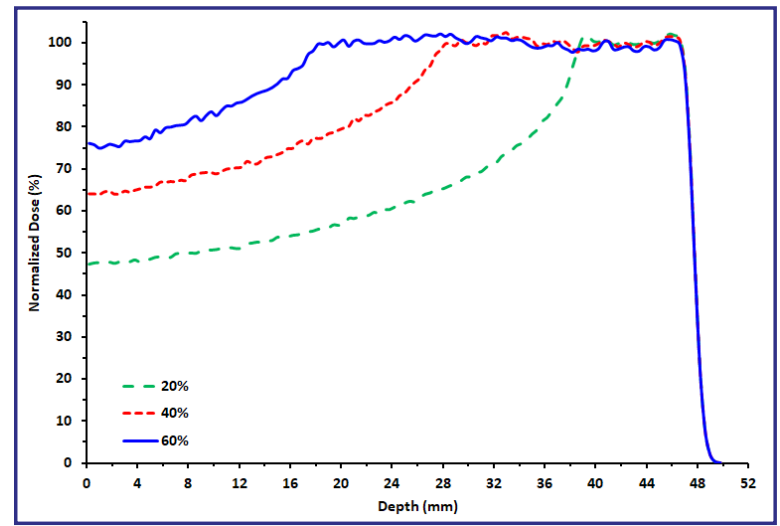

(d) $80 \mathrm{MeV}$

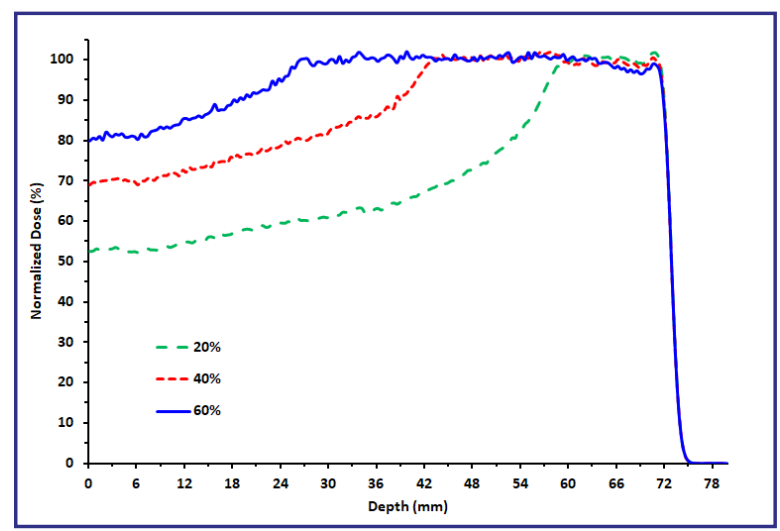

(f) $100 \mathrm{MeV}$

Fig. (4): SOBPs resulted from the newly designed RMWs. 
Table (3) : Simulated SOBPS.

\begin{tabular}{|c|c|c|c|c|c|c|c|}
\hline \multirow[b]{2}{*}{ Energy } & \multirow{2}{*}{$\begin{array}{c}\text { Designed } \\
\text { plateau size } \\
(\%)\end{array}$} & \multirow[b]{2}{*}{$\begin{array}{l}\text { Number } \\
\text { of steps }\end{array}$} & \multicolumn{4}{|c|}{ Resulting plateau } & \multirow{2}{*}{$\begin{array}{l}\text { Percentage } \\
\text { error }(\%)\end{array}$} \\
\hline & & & $\begin{array}{l}\text { Start } \\
(\mathrm{mm})\end{array}$ & $\begin{array}{l}\text { End } \\
(\mathrm{mm})\end{array}$ & $\begin{array}{c}\text { Size } \\
(\mathrm{mm})\end{array}$ & $\begin{array}{l}\text { Size } \\
(\%)\end{array}$ & \\
\hline \multirow{3}{*}{$50 \mathrm{MeV}$} & 20 & 6 & 14.55 & 17.85 & 3.3 & 18.5 & 7.60 \\
\hline & 40 & 11 & 10.85 & 17.85 & 7.0 & 39.2 & 1.96 \\
\hline & 60 & 16 & 7.15 & 17.85 & 10.7 & 59.9 & 0.09 \\
\hline \multirow{3}{*}{$60 \mathrm{MeV}$} & 20 & 6 & 21.3 & 26.1 & 4.8 & 18.4 & 8.05 \\
\hline & 40 & 12 & 15.3 & 26.1 & 10.8 & 41.4 & 3.45 \\
\hline & 60 & 18 & 9.3 & 26.1 & 16.8 & 64.4 & 7.28 \\
\hline \multirow{3}{*}{$70 \mathrm{MeV}$} & 20 & 6 & 29.3 & 35.7 & 6.4 & 17.92 & 10.4 \\
\hline & 40 & 12 & 21.1 & 35.7 & 14.6 & 40.89 & 2.24 \\
\hline & 60 & 18 & 13.5 & 35.7 & 22.2 & 62.2 & 3.64 \\
\hline \multirow{3}{*}{$80 \mathrm{MeV}$} & 20 & 6 & 38.6 & 46.6 & 8.0 & 17.2 & 14.0 \\
\hline & 40 & 13 & 27.8 & 46.6 & 18.8 & 40.34 & 0.85 \\
\hline & 60 & 19 & 17.8 & 46.6 & 28.8 & 61.8 & 3.00 \\
\hline \multirow{3}{*}{$90 \mathrm{MeV}$} & 20 & 6 & 48.6 & 58.6 & 10.0 & 17.06 & 14.7 \\
\hline & 40 & 13 & 34.6 & 58.6 & 24.0 & 40.95 & 2.39 \\
\hline & 60 & 19 & 22.6 & 58.6 & 36.0 & 61.43 & 2.39 \\
\hline \multirow{3}{*}{$100 \mathrm{MeV}$} & 20 & 8 & 57.8 & 71.4 & 13.6 & 19.05 & 4.76 \\
\hline & 40 & 16 & 41.8 & 71.4 & 29.6 & 41.5 & 3.64 \\
\hline & 60 & 24 & 26.2 & 71.4 & 45.2 & 63.3 & 5.5 \\
\hline
\end{tabular}

\section{CONCLUSIONS}

In this work, a comparison between the experimental SOBP for the proton beam in the CATANA facility and the simulated SOBP in the GATE application by using the gamma index criteria was performed to validate the used MC model. According to this study, the gamma passing rate $(\sim 98 \%)$ showed satisfactory agreement between the measurements and the simulations. New RMWs were designed to be used with different proton energies (ranges) and plateau sizes. The SOPBs of the newly designed RMWs also gave very acceptable results, and therefore, the GATE Monte Carlo application was demonstrated to be an excellent candidate for the simulation of passive scattering beamline systems in proton therapy.

\section{REFERENCES}

- World Health Organization (2018): Cancer key facts, https://www.who.int.

- Cancer treatment centers of America (2019): https://www.cancercenter.com.

- Parkin, D.M.; Bray, F. and Ferlay, J. (2005): Global cancer statistics. Cancer J. Clin, 55(2):74.

- Yajnik, S. (2013): Proton Beam Therapy: How Protons are Revolutionizing Cancer Treatment. Springer, New York.

- Vassiliev, O. (2017): Monte Carlo Methods for Radiation Transport, Springer, Switzerland.

- Agostinelli, S.; Allison, J. and Amako, K. (2003): Geant4 - A simulation Toolkit. Nucl. Inst. Meth, 506A: 250. 
- Visvikis, D.; Bardies, M. and Chiavassa, S. (2006): Use of the GATE Monte Carlo Package for Dosimetry Applications. Nucl. Inst. Meth, 569A:335.

- Nikjoo, H.; Uehara, S. and Emfietzoglou, D. (2012): Interaction of Radiation with Matter. CRC Press, USA.

- Particle therapy Co-Operative group (2019): https://www.ptcog.ch.

- Bijan, S.; Romano, F. and Cuttone, G. (2016): Designing a range modulator wheel to spread-out the Bragg peak for a passive proton therapy facility. Nucl. Instr. Meth. Phys. Res., 806A:101.

- Physics Lists EM constructors in Geant4 (2018): https://geant4.web.cern.ch/node/146.

- Grevillot, L.; Zahra, N. and Frisson, T. (2010): Optimization of GEANT4 settings for Proton Pencil
Beam Scanning simulations using GATE. Med. Phys, 268(20):3295.

- Brun, R. and Rademakers, F. (1997): ROOT - An Object Oriented Data Analysis Framework. AIHENP'96 Workshop Proceedings, Lausanne, Nucl. Inst. \& Meth. in Phys. Res, 389A: 8.

- Grcar, G. (2011): Mathematicians of Gaussian Elimination, Notices of the American Mathematical Society, 58(6):782.

- Stopping power and range tables of protons (2018): https://physics.nist.gov/PhysRefData/Star/ Text/PSTAR.html.

- Low, D.A.; Harms, W.B. and Mutic, S. (1998): A Technique for the Quantitative Evaluation of Dose Distributions. Med. Phys, 25:656. 